\title{
Effect of the Go4it multidisciplinary group treatment for obese adolescents on health related quality of life: a randomised controlled trial
}

Geesje H Hofsteenge ${ }^{1 *}$, Peter JM Weijs ${ }^{1,2,3}$, Henriette A Delemarre-van de Waal ${ }^{4,5}$, Maartje de Wit $^{6}$ and Mai JM Chinapaw ${ }^{2,7}$

\begin{abstract}
Background: We developed a Dutch outpatient multidisciplinary group treatment (Go4it) for obese adolescents, including cognitive behavioural therapy and education on healthy dietary and physical activity behaviour. This study examined the effect of Go4it on Health Related Quality of Life (HRQoL).

Methods: At our outpatient paediatric obesity clinic, obese adolescents ( $n=122,11-18$ years) were randomly assigned to 1) Go4it, 7 sessions with an interval of 2 weeks or 2) current regular care consisting of referral to a dietician in the home care setting (controls). Linear mixed model analysis was performed to evaluate the intervention effects on HRQoL at 6 and 18-month follow-ups. HRQoL indicators included the Child Health Questionnaire, the Paediatric Quality of Life Inventory ${ }^{\mathrm{TM}}$ Version 4.0 (PedsQL ${ }^{\mathrm{TM}} 4.0$ ), and the Body Esteem Scale (BES).

Results: In total, 95 adolescents (Go4it 57, controls 38) were included in the current analysis with a mean age of $14.5 \pm 1.7$ and mean BMI-SDS of $2.9 \pm 0.5$. At baseline, all participants experienced lower levels of physical and psychosocial well-being compared to a normal weight reference group. At the 18 month follow-up, we found small but beneficial intervention effects on all subscales of the PedsQL ${ }^{\mathrm{Tm}} 4.0$ and BES questionnaires. Two subscales improved significantly; i.e., physical health (between group difference $5.4 ; 95 \% \mathrm{Cl}: 0.3 ; 10.6$ ), and school functioning (between group difference $7.4 ;$ 95\%Cl: 1.6; 13.2).
\end{abstract}

Conclusion: Obese adolescents experienced lower HRQoL than their healthy peers. The Go4it intervention had small beneficial effects on HRQoL compared to the current regular care practices for obese adolescents.

Trial registration: Netherlands Trial Register: ISRCTN27626398, METC number: 05.134 (WMO, monocenter).

Keywords: Adolescents, Obesity, Health related quality of life, Randomized controlled trial

\section{Background}

Obesity is one of the most common chronic disorders in children and adolescents and its prevalence continues to increase rapidly. Of Dutch children aged 2-21 years old, $13-15 \%$ were overweight and $2 \%$ were classified as obese in 2009 [1]. Obesity is also one of the most stigmatizing and least socially accepted conditions in childhood [2]. The most widespread consequences of adolescent obesity are psychosocial [3]. Early adolescence may be a

\footnotetext{
* Correspondence: A.Hofsteenge@vumc.nl

'Department of Nutrition \& Dietetics, Internal Medicine, VU University Medical Center Amsterdam, De Boelelaan 1117, Amsterdam, HV 1081, The Netherlands

Full list of author information is available at the end of the article
}

particularly vulnerable period for reductions in Health Related Quality of Life (HRQoL) in overweight/obese youth, since heightened awareness of social exclusion and participation limitations occur [4,5]. As in a not overweight population there are adolescents with a higher or lower quality of life (QoL).

The World Health Organization defines QoL as "individuals' perceptions of their position in life in the context of the culture and value systems in which they live and in relation to their goals, expectations, standards and concerns [6]". It incorporates the person's physical health, psychological state, level of independence, social relationships, and their relationship to salient features of their environment in a complex way [6]. 
Negative self-image and a reduced self-esteem in overweight children, which can begin as early as the age of five, can ultimately result in sadness, loneliness, nervousness and high-risk behaviour's as children develop into obese adolescents [7]. Obesity is also predictive of being the victim of bullying in adolescents [8]. These widespread psychosocial consequences of childhood obesity in adolescents impair their HRQoL [2]. Schwimmer et al. showed that obese children and adolescents (5-18 years old), who where newly referred to the clinic, reported significantly lower QoL in all domains compared with normal weight children and adolescents [2].

Notwithstanding the high prevalence of child obesity, little evidence exists regarding effective child obesity treatments [9]. Most studies included children age 712 years old, and only a few studies have evaluated treatment of adolescent obesity [9-12]. Often, outpatient treatment for the obese is focused on nutrition education and physical activity [9-13]. Wille et al. [14] and Vignolo et al. [15] showed improvement on the effect of their inpatient and outpatient treatment programs not only on diet and physical activity but also on the HRQoL of children aged 6-16 and 6-12 years, respectively. Breat et al. showed promising results of cognitive behavioural modification techniques regarding lifestyle changes in obese children [16]. Based on these positive experiences, and the fact that there is no effective treatment available for this age group, we developed a multidisciplinary group treatment for obese Dutch adolescents (Go4it) [17]. Understanding HRQoL can contribute to a better awareness of the patients' needs, as well as improve care and treatment.

This study describes the long-term effects of the Go4it group treatment for obese adolescents on HRQoL aspects in a randomised controlled trial. We hypothesised that at baseline our study sample would have lower HRQoL compared to a normal weight reference group. We also hypothesised that the Go4it treatment would have a beneficial effect on HRQoL.

\section{Methods}

\section{Subjects and design}

The present study is a randomised controlled trial evaluating the effect of the Go4it multidisciplinary group treatment for obese adolescents, on HRQoL at six and 18 month follow-ups. Adolescents were referred to the outpatient paediatric obesity clinic of the VU University Medical Center Amsterdam by their general practitioner or school physician. During the first visit one of three paediatric-endocrinologists interviewed all adolescents concerning their medical history, social problems, teasing, weight development, socio-economic status (SES) and ethnicity according to a standard protocol. Subjects were categorised as having a western ethnicity when both parents were Dutch or with at least one parent was born outside the Netherlands, but inside Europe (including former Yugoslavia and Soviet Union), North America, Oceania, Indonesia or Japan. Subjects with at least one parent born in Turkey, Africa, Latin America or Asia were classified as non-western [18]. The physical examination included height, weight, waist circumference, blood pressure and pubertal Tanner stage [19].

The subjects and their parents received an informational brochure about the study. Within two weeks, the research assistant checked their willingness to participate. Subjects were eligible when they met the following inclusion criteria: 1) age 11-18 years; and 2) overweight or obese according to the definition of Cole et al. with gender and age specific cut off values [20]. Exclusion criteria were: not Dutch-speaking, obesity as a result of a known syndrome or organic cause (hypothyroidism), mental retardation, physical limitations (e.g. crutches or wheelchair) and diagnosis of type 2 diabetes mellitus. The research assistant used block randomisation to assign subjects randomly to the intervention $(60 \%)$ or control group (40\%), using SPSS for random selection. This distribution was chosen to recruit a sufficient number of adolescents to start the intervention sessions. Randomisation was stratified for sex and age group (11-14 years old and 15-18 years old). Since adolescents knew to which group they were assigned, participants could not be blinded. The medical ethical committee of VU University Medical Center Amsterdam approved the protocol. Adolescents as well as their parents gave written informed consent.

\section{Intervention}

Go4it is a multidisciplinary group treatment for obese adolescents based on the programs of Braet et al. [16], Epstein et al. [21], and the educational materials of the Dutch Obesity Intervention in Teenagers (DOiT) [22]. During 7 sessions with two-week intervals, the adolescents received education on healthy dietary behaviour, screen behaviour and physical activity. The group size was 8 to 12 adolescents. The first session was focussed on increasing awareness of the current lifestyle. Besides dietary and activity journals, step counters (pedometers) were used to increase awareness of the actual behaviour. Next, adolescents were instructed to set goals with respect to improving their physical activity and dietary behaviour. Additionally cognitive- behavioural therapy characteristics (mainly based on problem-solving techniques) were used, for example, learning how to improve their lifestyle, learning to cope with teasing, to improve self-esteem, and how to maintain energy balance. Go4it works with homework tasks and the education is interactive. Go4it was carried out in an outpatient clinic involving a dietician, psychologist, and a paediatric-endocrinologist. They were also all involved in the development of Go4it. 
In addition, two separate parallel sessions for parents were organised corresponding to the first and fourth session of the adolescents. These parental sessions consisted of education concerning healthy dietary behaviour and physical activity, the health risks of overweight and how to support their obese children in improving their behaviour. Four booster group sessions for the adolescents were scheduled 6, 14, 26, and 36 weeks after the 3-months intervention period, in order to encourage the adolescents to maintain or further improve their energy balance behaviour, discuss problems and answer questions. Throughout the program the adolescents remained in the same peer group. Special materials were developed for this program: an information book, a workbook, and a dietary and activity diary. In addition, specific worksheets for every session were developed. The control group received the current regular care in the Netherlands, consisting of referral to a dietician in the home care setting. Adolescents had to make an appointment themselves. Reasons for non-compliance were collected by phone and questionnaire. Details of the intervention have been published elsewhere [17].

\section{Study protocol}

After an overnight fast, subjects attended the outpatient clinic. Height was measured with an accuracy of $0.1 \mathrm{~cm}$ with an electronic stadiometer (KERN 250D, De Grood Metaaltechniek, Nijmegen, The Netherlands). Body weight was measured (in underwear) within $0.1 \mathrm{~kg}$ with a calibrated electronic flat scale (SECA 861, Schinkel, Nieuwegein, The Netherlands). Weight and height were used to calculate BMI $\left(\mathrm{kg} / \mathrm{m}^{2}\right)$. For calculation of BMI standard deviation scores (BMI-SDS) or z-scores, a reference database of Dutch children was used (www. growthanalyser.org; version 3.5). The researcher conducted all measurements. The adolescents filled in the questionnaires (PedsQL ${ }^{\mathrm{m}} 4.0, \mathrm{CHQ}$ and the BES) in the morning during a visit to the obesity clinic. The adolescents completed the questionnaires' independently and the research assistant entered the data. Baseline measurements took place between November 2006 and August 2008. Measurements were repeated after 6 and 18 months.

\section{Questionnaires}

HRQoL was examined using the generic reliable and validated Paediatric Quality of Life Inventory ${ }^{\mathrm{mm}}$ Version 4.0 (PedsQL ${ }^{\mathrm{mm}} 4.0$ ) $[23,24]$ and the generic Child Health Questionnaire (CHQ) [25]. PedsQL ${ }^{\text {ma }} 4.0$ assesses physical, emotional, social and school functioning, while $\mathrm{CHQ}$ assesses physical, behavioural, mental and social functioning.

The 23-item PedsQL ${ }^{\mathrm{mm}} 4.0$ questionnaire encompasses physical functioning (eight items), emotional functioning (five items), social functioning (five items) and school functioning (five items). A 5-point Likert scale was used for response $(0=$ never a problem; $4=$ almost always a problem). Items are reversed scored and linearly transformed to a 0-100 scale, so that higher scores indicate better HRQoL. A total scale score and physical and psychosocial health summary scores were also calculated $[23,25]$.

The psychometric properties of the Dutch translation of the PedsQL ${ }^{\mathrm{mm}} 4.0$ questionnaires has been well established by van Engelen et al. [26]. Their study population consisted of 496 healthy schoolchildren aged 57 years $(n=92), 8-12$ years $(n=219)$ and $13-18$ years $(\mathrm{n}=185)$. There are no known data for weight or BMI [26]. Reliability of the PedsQL ${ }^{\mathrm{m} m} 4.0$ was good in our sample (Total score: Cronbach's $\alpha=0.87$; Physical health score: Cronbach's $\alpha=0.79$; Psychosocial health score: Cronbach's $\alpha=0.81$ ).

The CHQ Child Form 87 questionnaire encompasses 12 domains of which each item contains 4,5 or 6 response alternatives. Per scale, the items are summed up (some recoded/recalibrated) and transformed to a 0 (worst possible score) to 100 (best possible score) scale [25]. In this study, a physical summary scale was computed, as the mean of the items in the CHQ-subscales of physical functioning, role/social limitations-physical, general health perceptions and bodily pain. Further, we computed a psychosocial summary scale, as the mean of the items in the CHQ-subscales of role/social limitationsemotional, role/social limitations-behavioural, self esteem, mental health and general behaviour. The psychometric properties of the Dutch translation of the CHQ questionnaire has been well established in a population of 466 schoolchildren (age 9-17 years) by Raat et al. [27]. In these children, allergies (17\%), eczema (8\%), migraines (6\%) and asthma (5\%) were the most prevalent reported conditions. Again, there is no known information for weight or BMI [27]. Reliability of both the physical and psychosocial summary scales was good in our sample (Cronbach's $\alpha=0.81$ and 0.93).

\section{Body esteem scale}

Body esteem is an essential part of psychosocial wellbeing in overweight adolescents, but not covered in PedsQL ${ }^{r m} 4.0$ nor CHQ. The Body Esteem Scale (BES) is a validated questionnaire [28] on general feelings about appearance, weight satisfaction and evaluations of attributions to others about one's body and appearance. Mean scores range from 0 (worst possible score) to 4 (best possible score), with higher scores representing a better body esteem.

\section{Sample size}

The sample size calculation is based on the primary outcome of the trial i.e. BMI-SDS: the number of participants 
needed to detect a difference in BMI-SDS of 0.29 (10\%) between the intervention and control group after 18 months with a standard deviation of 0.5 is 43 subjects per group with an alpha of .05 and a power of .80. A sample of 108 persons ( $n=54$ per group) was required taking into account a dropout rate of $25 \%$.

\section{Statistical analysis}

Baseline characteristics were analysed by t-tests for continuous variables and Chi-square tests for categorical variables. Scoring and substitution of missing values was performed according to existing manuals. In the case of $50 \%$ or less missing per subscale, substitution by the mean was used $[24,25,28]$. Subscales with higher amounts of missing values were considered missing resulting in a varying amount of participants included in the analyses. Group comparisons were performed according the intention-to-treat principle whereby all subjects were analysed in the group to which they were initially assigned. Linear mixed models were applied to assess the effect of the intervention over time. A random intercept and a random slope with time were assumed. Age-, sex- and, ethnicity adjusted analyses were performed with intervention as the categorical variable and time as the continuous variable; an interaction term for intervention and time was also included. B coefficients (between group difference), 95\% confidence intervals and $p$ values were calculated. This approach has increased statistical power as it accounts for within-person correlation over time and allows different numbers of assessments. All assessments, including baseline, were taken into account. A $p$ value of $<0.05$ was considered statistically significant. Effect modification by sex, age and ethnicity was checked by adding an interaction term between group allocation and the potential moderator. For effect modification, a $p$ value of $<0.1$ was considered statistically significant. Finally, effect size estimations (Cohen's $d$ ) were calculated in order to decide whether statistical differences were clinically relevant. Effect sizes relate to the difference in mean scores to the dispersion of the scores: [Mean baseline - Mean follow-up]/pooled standard deviation [29]. Following Cohen's $d$ effect size, $d=0.2$ was taken to

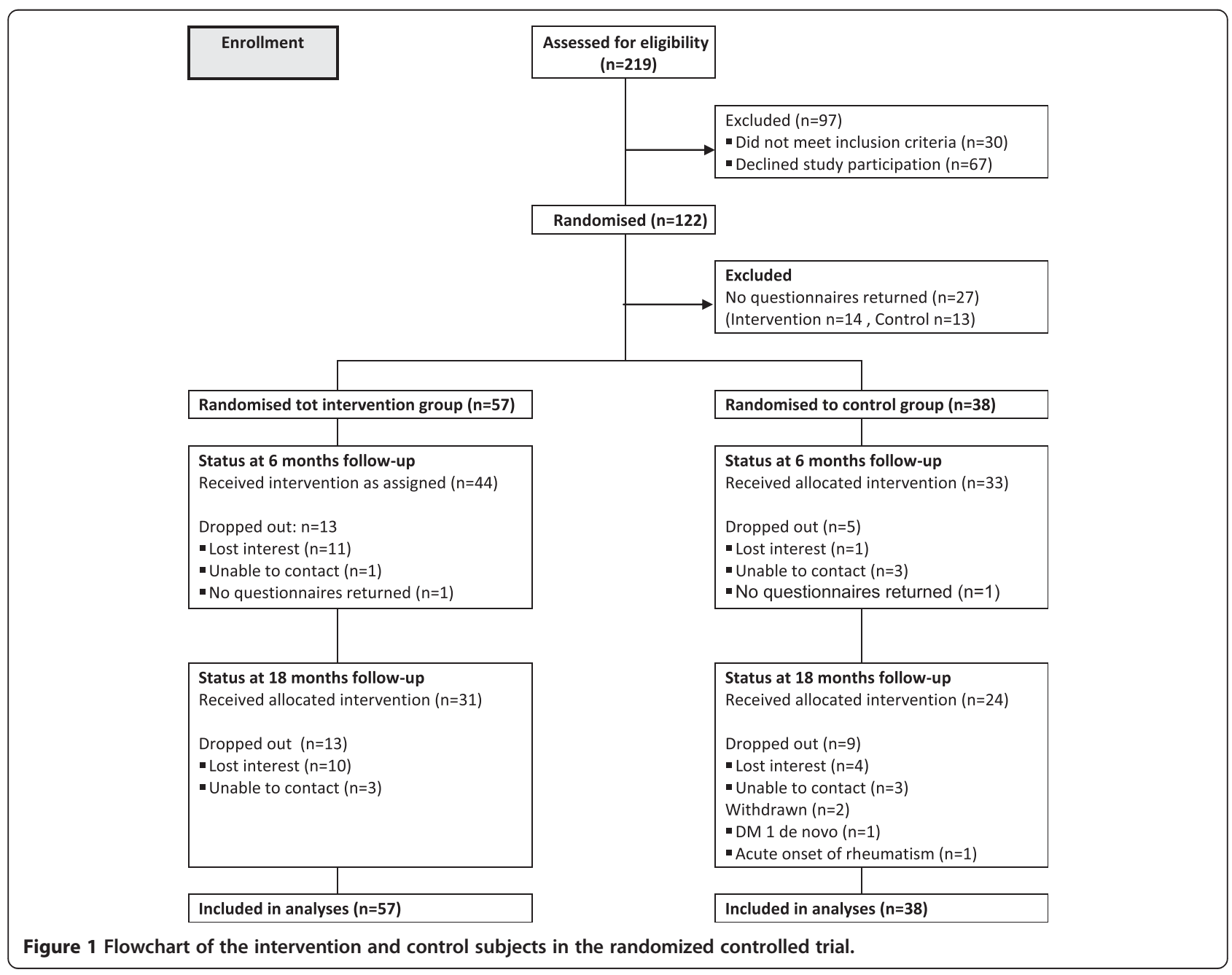


indicate a small effect size, $d=0.5$ a moderate effect size, and $d=0.8$ a large effect size [30]. All analyses were performed using SPSS software (version $18 \cdot 0,2009$ SPSS Inc., Chicago, Illinois, USA).

\section{Results}

Subjects

Figure 1 shows the consort diagram for the Go4it trial. Of the 219 adolescents who were assessed for eligibility, 122 consented to the trial and were randomly $(60: 40)$ assigned to the intervention $(\mathrm{n}=71)$ and control group $(\mathrm{n}=51)$. At 18 months two subjects from the control group were excluded from the analyses, one developed type 1 diabetes and another was diagnosed with acute rheumatism. Linear mixed models were applied whereby all subjects were analyzed in the group to which they were randomly assigned.

Of the 122 randomised adolescents, 95 completed all questionnaires at baseline. Table 1 presents anthropometric and demographic characteristics of these 95 study participants. No significant differences were found in baseline characteristics between Go4it and control group, nor between the 95 study participants and the 27 adolescents who did not fill in the baseline questionnaires.

\section{Physical and psychosocial well being}

Physical and psychosocial well-being scores and between group differences at 6 and 18 month follow-ups are presented in Table 2. At baseline, participants experienced lower levels of physical and psychosocial well-being scores compared with a healthy reference group from Engelen et al. (PedsQL ${ }^{\mathrm{mm}} 4.0$ ) [26] and Raat et al. (CHQ) [27].

At the 18 month follow-up we found small but beneficial intervention effects on all subscales of the PedsQL ${ }^{\mathrm{mm}} 4.0$ and BES questionnaires. Two subscales of the PedsQL $\mathrm{L}^{\mathrm{m}} 4.0$ improved significantly, i.e. physical health (between group difference 5.4; 95\% CI: 0.3; 10.6) with a clinical effect size of 0.7 , and school functioning (between group difference $7.4 ; 95 \%$ CI: 1.6; 13.2), with a clinical effect size of 0.3 . We found effect modification by sex and ethnicity on a few HRQoL outcomes. The results were inconsistent and for this are reason not presented.

Concerning compliance, 42 (59\%) of the 71 subjects in the intervention group, attended at least five Go4it sessions. The reasons for not attending the Go4it sessions included lack of motivation to change dietary habits, lack of belief of parents in their child's potential success to lose weight, previous unsuccessful dieting experiences, travel distance and the limited time of working parents and schoolchildren. Seven of the 29 subjects who attended less than five sessions never started the Go4it program. At 6 months, 21 (48\%) subjects in the control group had never visited a dietician, 4 subjects visited a dietician once,
Table 1 Baseline demographic and anthropometric characteristics of Go4it and control group participants

\begin{tabular}{|c|c|c|c|}
\hline & $\begin{array}{l}\text { Intervention group } \\
(n=57)\end{array}$ & $\begin{array}{l}\text { Control group } \\
(n=38)\end{array}$ & $p$ \\
\hline Age, $y^{1}$ & $14.6(1.6)$ & $14.5(1.7)$ & $0.8^{a}$ \\
\hline \multicolumn{4}{|l|}{$\operatorname{Sex}(n)$} \\
\hline Girls/Boys & $29 / 28$ & $24 / 14$ & $0.2^{a}$ \\
\hline Height, cm & $168.4(10.1)$ & $165.3(8.3)$ & $0.1^{a}$ \\
\hline Weight, kg & $94.6(18.6)$ & 91.9 (17.9) & $0.5^{a}$ \\
\hline $\mathrm{BMI}, \mathrm{kg} / \mathrm{m} 2$ & $33.1(4.4)$ & $33.4(5.0)$ & $0.8^{a}$ \\
\hline BMI-SDS & $2.9(0.4)$ & $2.9(0.5)$ & $0.8^{a}$ \\
\hline \multicolumn{4}{|l|}{ Ethnicity (n) } \\
\hline Western/Non-western & $29 / 28$ & $14 / 24$ & $0.2^{b}$ \\
\hline \multicolumn{4}{|l|}{ Teasing (n) } \\
\hline Yes/No & $23 / 32$ & $17 / 18$ & $0.5^{b}$ \\
\hline Missing $^{2}$ & 3 & 3 & \\
\hline \multicolumn{4}{|c|}{ Education adolescents (n) } \\
\hline Low $^{3}$ & 44 & 28 & $0.7^{b}$ \\
\hline $\mathrm{High}^{4}$ & 12 & 9 & \\
\hline Missing $^{2}$ & 1 & 1 & \\
\hline
\end{tabular}

${ }_{1}^{1}$ Data are (means (sd)), unless otherwise indicated.

${ }^{2}$ No information available on item in question, ${ }^{\mathrm{a}} t$-test, ${ }^{\mathrm{b}} \mathrm{Chi}$ Square.

${ }^{3}$ primary school, or lower secondary education,

4 upper secondary education.

6 twice, 7 three or more times, and for 6 subjects it is unknown. The main reported reason for not making an appointment was lack of motivation because of previous unsuccessful dieting experiences with or without a dietician [31].

\section{Discussion and conclusions}

This study describes the effectiveness of an obesity treatment program on health related quality of life (HRQoL) aspects in obese adolescents. We found small but beneficial intervention changes on all subscales of the PedsQL ${ }^{\mathrm{Tm}} 4.0$ and BES questionnaires. Two subscales of the PedsQL ${ }^{\mathrm{mm}} 4.0$, physical health and school functioning, improved significantly in favour of the intervention group. Thus, our low intensive outpatient multidisciplinary group treatment had small but beneficial effects on quality of life of obese adolescents.

\section{Implications}

As we mentioned earlier few studies examined HRQoL among obese adolescents enrolled in outpatient programs and the effects of these programs on HRQoL [14-16]. Most programs targeting obese adolescents mainly focus on weight change $[9,13]$.

In our study we found a beneficial decrease in BMISDS at the 6 month follow-up (between group difference: 
Table 2 CHQ- CF87 scale -, PedsQL ${ }^{\mathrm{Tm}} 4.0$ and BES mean scores and between group difference between the intervention group and control group at baseline, 6 month and 18 month follow-ups

\begin{tabular}{|c|c|c|c|c|c|c|c|c|}
\hline & \multicolumn{3}{|c|}{ Intervention group ${ }^{2}$} & \multicolumn{5}{|c|}{ Control group ${ }^{2}$} \\
\hline & Baseline & 6 month & 18 month & Baseline & 6 month & 18 months & 6 months & 18 months \\
\hline & Mean $\pm s d$ & Mean $\pm s d$ & Mean $\pm s d$ & Mean $\pm s d$ & Mean $\pm s d$ & Mean $\pm s d$ & B $(95 \% \mathrm{Cl})^{3}$ & B $(95 \% \mathrm{Cl})$ \\
\hline \multicolumn{9}{|l|}{$\mathrm{CHQ}^{-\mathrm{CF} 87^{1}}$} \\
\hline Physical summary scale & $78.2 \pm 12.1$ & $80.2 \pm 11.2$ & $82.2 \pm 10.4$ & $78.3 \pm 11.1$ & $82.5 \pm 9.9$ & $80.4 \pm 11.6$ & $-2.0(-6.6 ; 2.5)$ & $2.3(-2.9 ; 7.4)$ \\
\hline Psychosocial summary scale & $80.9 \pm 10.1$ & $82.6 \pm 10.9$ & $85.1 \pm 9.9$ & $81.5 \pm 10.0$ & $83.9 \pm 8.3$ & $83.5 \pm 10.2$ & $-1.5(-5.9 ; 2.8)$ & $1.0(-4.0 ; 6.0)$ \\
\hline \multicolumn{9}{|l|}{ PedsQL ${ }^{\mathrm{tm}} 4.0^{1}$} \\
\hline Total score & $75.1 \pm 12.2$ & $78.5 \pm 11.2$ & $81.7 \pm 12.0$ & $75.7 \pm 10.7$ & $77.9 \pm 10.0$ & $77.2 \pm 10.5$ & $-0.1(-3.5 ; 3.3)$ & $3.8(-0.2 ; 7.7)$ \\
\hline Physical health & $76.5 \pm 14.8$ & $83.1 \pm 11.8$ & $86.7 \pm 11.8$ & $76.4 \pm 13.2$ & $78.6 \pm 11.9$ & $79.8 \pm 11.4$ & $3.6(-1.0 ; 8.2)$ & $5.4(0.3 ; 10.6)^{4}$ \\
\hline Psychosocial health & $74.7 \pm 12.6$ & $76.9 \pm 12.0$ & $80.0 \pm 12.8$ & $75.4 \pm 11.2$ & $77.8 \pm 10.2$ & $76.4 \pm 11.6$ & $-1.0(-4.6 ; 2.6)$ & $3.4(-0.8 ; 7.6)$ \\
\hline Emotional functioning & $74.7 \pm 17.2$ & $77.9 \pm 15.3$ & $76.1 \pm 17.9$ & $74.5 \pm 15.8$ & $76.1 \pm 12.0$ & $76.1 \pm 16.4$ & $1.5(-4.1 ; 7.1)$ & $1.8(-4.8 ; 8.4)$ \\
\hline Social functioning & $78.2 \pm 15.5$ & $81.8 \pm 14.0$ & $87.3 \pm 12.4$ & $78.8 \pm 16.1$ & $82.8 \pm 14.0$ & $81.3 \pm 13.9$ & $-0.5(-5.1 ; 4.1)$ & $2.4(-3.0 ; 7.8)$ \\
\hline School functioning & $71.1 \pm 15.3$ & $71.1 \pm 15.0$ & $76.6 \pm 16.2$ & $73.0 \pm 15.4$ & $75.5 \pm 15.3$ & $71.7 \pm 14.4$ & $-3.2(-8.1 ; 1.7)$ & $7.4(1.6 ; 13.2)^{5}$ \\
\hline \multicolumn{9}{|l|}{$\mathrm{BES}^{1}$} \\
\hline Body appearance & $1.8 \pm 0.7$ & $2.1 \pm 0.6$ & $2.2 \pm 0.7$ & $1.9 \pm 0.8$ & $2.1 \pm 0.7$ & $2.2 \pm 0.7$ & $0.0(-0.2 ; 0.3)$ & $0.1(-0.1 ; 0.4)$ \\
\hline Weight satisfaction* & $1.6 \pm 0.7$ & $1.7 \pm 0.7$ & $1.7 \pm 0.7$ & $1.7 \pm 0.6$ & $1.8 \pm 0.7$ & $1.8 \pm 0.7$ & $0.0(-0.2 ; 02)$ & $0.2(0.0 ; 0.5)$ \\
\hline Body attribution & $1.8 \pm 0.8$ & $2.1 \pm 0.7$ & $2.3 \pm 0.8$ & $1.9 \pm 1.0$ & $1.9 \pm 0.7$ & $2.0 \pm 0.6$ & $(0.0 ; 0.5)$ & $0.3(0.0 ; 0.6)$ \\
\hline
\end{tabular}

${ }^{1} \mathrm{CHQ}$ scores and PedsQL ${ }^{\mathrm{TM}} 4.0$ range from 0 (worst possible score) to 100 (best possible score) with higher scores representing a better quality of life. BES scores range from 0 (worst possible score) to 4 (best possible score), with higher scores representing a better body esteem.

${ }^{2}$ The numbers of subjects varied at 6 months from 44-45 in the intervention group and from 33-34 in the control group. After the 18 month follow-up the numbers of subjects varied in the intervention group between 34-35 and in the control group from 23-28.

${ }^{3}$ As indicated by unstandardised regression coefficients (B) and $95 \%$ confidence intervals $(95 \% \mathrm{Cl})$, adjusted for baseline value, age, sex and ethnicity.

${ }^{4}=$ effect size d: 0.7,

${ }^{5}=$ effect size d: 0.3 .

$-0.10(-0.23 ; 0.04)$ and a significant decrease at the 18 month follow-up (between group difference: -0.16; 95\% CI: -0.30;-0.02) [31]. We found a significant but low correlation $(\mathrm{R}=-.359)$ between change in BMI-SDS and change in physical health (PedsQL ${ }^{\mathrm{ms}} 4.0$ ) at the 6 month follow-up, but not at 18 month follow-up.

We also found a significant effect on experienced physical health and school functioning at the 18 month follow up. A possible reason for the improved school functioning may be the cognitive behavioural therapy elements of Go4it focussing on dealing with difficult situations and how to react to teasing. This may have provided them with tools for less distraction and better concentration at school. The improved physical health may also have resulted in fewer days of sick leave and thereby had a positive influence on school attendance. As the CHQ does not tap into school issues, we found no differences there.

\section{Other studies}

Our study is in line with previous studies showing that all mean physical and psychosocial summary scores at baseline were lower in obese adolescents in comparison with healthy peers. Therefore, they experience a lower quality of life $[23,26]$, but similar to those reported in other obese samples [23,32]. In a cross-sectional study, de Beer et al. compared HRQoL in obese Dutch adolescents to age and sex matched normal weight controls $(n=62)$ [32]. CHQ and PedsQL ${ }^{\mathrm{rm}} 4.0$ scores of obese adolescents in their sample were less than their normal weight controls and resembled the mean scores in our sample. Also similar scores on both questionnaires' were found among obese children aged 8-18 years old and among Dutch children suffering form a chronic health condition to those in our sample [26,33].

For the BES, Dutch reference data on obese adolescents are currently not available. In a study by Mendelson et al. [28], in which body esteem among healthy Canadian adolescents was measured, adolescents reported mean scores ranging from 1.8 to 3.0. As expected, obese adolescents in our study reported slightly lower mean body esteem scores.

Unlike the two subscales of the PedsQL ${ }^{\mathrm{Tm}} 4.0$, we found no significant intervention effect on the CHQ. The PedsQL ${ }^{\mathrm{Tm}} 4.0$ addresses more serious problems and includes more subscales than the CHQ. The PedsQL ${ }^{\mathrm{rm}} 4.0$ may therefore be more sensitive to subtle changes.

\section{Strength and limitations}

Most outpatient programs in adolescents that combine education on healthy nutrition and physical activity with cognitive behavioural therapy primarily evaluated effects on weight status. Few have evaluated effects on HRQoL. 
Other strengths of our study are the randomised controlled trial design, the relatively easy to implement outpatient intervention and the relatively long-term follow up. Another strength is the information from three questionnaires, providing the opportunity to examine intervention effects on various aspects of HRQoL.

A limitation of our study may be selection bias, because participants were obese adolescents referred to a medical obesity outpatient clinic in Amsterdam. Our findings may not be generalisable to the larger group of obese adolescents seen or treated by general health practitioners in smaller cities in the Netherlands. The majority (59\%) of our study sample was living in Amsterdam, where almost $50 \%$ of the population is of non-western descent, this is in contrast to other regions of the Netherlands where generally $30 \%$ of the population is from Non-Western descent (www.zorgatlas.nl). In our study sample only $34 \%$ were of Dutch origin and the majority of the non-western adolescents were from Turkish descent. However, we found no differences in intervention effects between adolescents from different ethnicities. Moreover, our study sample consists of obese adolescents seeking treatment. These adolescents generally had a higher level of psychopathology than those not seeking treatment [34]. Elevated levels of psychopathology are related with impaired HRQoL [35].

Another limitation is that our study had insufficient power to detect a significant difference in HrQol, since the power calculation was based on the primary outcome i.e. BMI-SDS. Therefore we focused on effect estimates and confidence intervals rather than statistical significance. Also a limitation of our trial is the low adherence to the Go4it program. Many adolescents were not motivated to attend the Go4it sessions every other week. Even after signing the informed consent form, some adolescents and their families were not willing to participate. We encouraged participant compliance by sending reminders using text messages and phone contact one week before sessions. The main reasons for not attending the Go4it sessions were the travel distance and the limited time of working parents and schoolchildren. Therefore, we recommend implementation of Go4it in a setting closer to the home environment, such as the child health care environment or school setting. The dropout rates of 20 and $44 \%$ at the 6 and 18-month follow-ups, respectively, are comparable to previous studies in obese adolescents (12-47\%) [13,36-39].

In summary, obese adolescents experienced lower quality of life than their healthy peers.

Our low intensity, multidisciplinary, outpatient group treatment, Go4it, had small but beneficial effects on the health related quality of life of obese adolescents. This study shows that an intervention program targeting a healthy lifestyle among obese adolescents can improve their quality of life.

\section{Ethical approval}

This study was approved by the Medical Ethical Committee of the VU University Medical Center Amsterdam. The adolescents as well as their parents gave written informed consent.

\section{Competing interests}

The authors declare that they have no competing (financial) interests.

\section{Authors' contributions}

$\mathrm{GH}, \mathrm{PW}, \mathrm{HD}, \mathrm{MW}$, and MC provided support in the design of the study and contributed to the main ideas of this paper. GH conducted the statistical analyses and drafted the manuscript. PW, MC, MW and HD provided support during the development and implementation of the intervention. MC, MW and PW provided critical comments on the analyses and manuscript. All authors contributed to the interpretation of data and drafting of the manuscript. All authors read and approved the final manuscript.

\section{Acknowledgements}

We acknowledge and express our gratitude to all the adolescents and parents who participated in the study, Claudet van Kampen, Suzanne ten Dam and Eartha Rodgers for their support and assistance during the collection and processing of the data of this research, and the outpatients clinic staff for their facilitative services.

\section{Funding}

This study was funded by The Netherlands Organisation for Health Research and Development (ZONMW) (no: 50-50110-98-255). The funding organization was not involved in the design and conduct of the study; nor were they involved in the collection, management, analysis, and interpretation of the data. Finally, the funding organization was not involved in the preparation, review or approval of the manuscript.

\section{Author details}

${ }^{1}$ Department of Nutrition \& Dietetics, Internal Medicine, VU University Medical Center Amsterdam, De Boelelaan 1117, Amsterdam, HV 1081, The Netherlands. ${ }^{2}$ EMGO Institute for Health and Care Research, VU University Medical Center Amsterdam, Amsterdam, The Netherlands. ${ }^{3}$ Department of Nutrition \& Dietetics, Amsterdam University of Applied Sciences, Amsterdam, The Netherlands. ${ }^{4}$ Department of Paediatrics, Leiden University Medical Center, Leiden, The Netherlands. ${ }^{5}$ Department of Paediatrics, VU University Medical Center Amsterdam, Amsterdam, The Netherlands. ${ }^{6}$ Department of Medical Psychology, EMGO Institute for Health and Care Research, VU University Medical Center Amsterdam, Amsterdam, The Netherlands. ${ }^{7}$ Department of Public and Occupational Health, VU University, Amsterdam, Amsterdam, The Netherlands.

Received: 9 July 2013 Accepted: 26 September 2013

Published: 8 October 2013

\section{References}

1. Schonbeck Y, Talma H, Van Dommelen P, Bakker B, Buitendijk SE, Hirasing RA, et al: Increase in prevalence of overweight in Dutch children and adolescents: a comparison of nationwide growth studies in 1980, 1997 and 2009. PLoS One 2011, 6:e27608.

2. Schwimmer JB, Burwinkle TM, Varni JW: Health-related quality of life of severely obese children and adolescents. JAMA 2003, 289:1813-1819.

3. Dietz WH: Health consequences of obesity in youth: childhood predictors of adult disease. Pediatrics 1998, 101:518-525.

4. Tsiros MD, Olds T, Buckley JD, Grimshaw P, Brennan L, Walkley J, et al: Health-related quality of life in obese children and adolescents. Int J Obes (Lond) 2009, 33:387-400.

5. Zametkin AJ, Zoon CK, Klein HW, Munson S: Psychiatric aspects of child and adolescent obesity: a review of the past 10 years. J Am Acad Child Adolesc Psychiatry 2004, 43:134-150.

6. Murphy B, Herrman H, Hawthorne G, Pinzone T, Evert H: WHOQOL-100, WHOQOL BREF and CA-WHOQOL INSTRUMENTS User's manual and interpretaion GuideThe world health organization quality of life (WHOQOL) study. Australian: The World Health Organization Quality of Life (WHOQOL) study; 2000. Ref Type: Generic. 
7. Pinhas-Hamiel O, Singer S, Pilpel N, Fradkin A, Modan D, Reichman B: Health-related quality of life among children and adolescents: associations with obesity. Int J Obes (Lond) 2006, 30:267-272.

8. Griffiths LJ, Wolke D, Page AS, Horwood JP: Obesity and bullying: different effects for boys and girls. Arch Dis Child 2006, 91:121-125.

9. Oude Luttikhuis H, Baur L, Jansen H, Shrewsbury VA, O'Malley C, Stolk RP, et al: Interventions for treating obesity in children. Cochrane Database Syst Rev 2009, 1:CD001872.

10. Epstein LH, Valoski A, Wing RR, McCurley J: Ten-year outcomes of behavioral family-based treatment for childhood obesity. Health Psychol 1994, 13:373-383.

11. Wilson P, O'Meara S, Summerbell C, Kelly S: The prevention and treatment of childhood obesity. Qual Saf Health Care 2003, 12:65-74.

12. Jelalian E, Saelens BE: Empirically supported treatments in pediatric psychology: pediatric obesity. J Pediatr Psychol 1999, 24:223-248.

13. Savoye M, Shaw M, Dziura J, Tamborlane W, Rose P, Guandalini C, et al: Effects of a weight management program on body composition and metabolic parameters in overweight children: a randomized controlled trial. JAMA 2007, 297:2697-2704.

14. Wille N, Erhart M, Petersen C, Ravens-Sieberer U: The impact of overweight and obesity on health-related quality of life in childhood-results from an intervention study. BMC Public Health 2008, 8:421.

15. Vignolo M, Rossi F, Bardazza G, Pistorio A, Parodi A, Spigno S, et al: Fiveyear follow-up of a cognitive-behavioural lifestyle multidisciplinary programme for childhood obesity outpatient treatment. Eur J Clin Nutr 2008, 62:1047-1057.

16. Braet $C$, Tanghe A, Decaluwe V, Moens E, Rosseel Y: Inpatient treatment for children with obesity: weight loss, psychological well-being, and eating behavior. J Pediatr Psychol 2004, 29:519-529.

17. Hofsteenge GH, Chinapaw MJM, Weijs PJM, Van Tulder MW, Delemarre-van de Waal HA: Go4it; study design of a randomised controlled trial and economic evaluation of a multidisciplinary group intervention for obese adolescents for prevention of diabetes mellitus type 2. BMC Public Health 2008, 8:410.

18. Statistics Netherlands (www.cbs.nl): Standaarddefinitie allochtonen. 10th edition. Voorburg, The Netherlands: Hoe doet het CBS dat nou? 2000:24-25.

19. Tanner JM: Growth and maturation during adolescence. Nutr Rev 1981 39(2):43-55.

20. Cole TJ, Bellizzi MC, Flegal KM, Dietz WH: Establishing a standard definition for child overweight and obesity worldwide: international survey. BMJ 2000, 320:1240-1243.

21. Epstein $L H$, Myers MD, Raynor HA, Saelens BE: Treatment of pediatric obesity. Pediatrics 1998, 101:554-570.

22. Singh AS, Chinapaw MJM, Kremers SPJ, Visscher TLS, Brug J, Mechelen van W: Design of the dutch obesity intervention in teenagers (NRG-DOiT): systematic development, implementation and evaluation of a school-based intervention aimed at the prevention of excessive weight gain in adolescents. BMC Public Health 2006, 6:304.

23. Varni JW, Seid M, Kurtin PS: PedsQL 4.0: reliability and validity of the pediatric quality of life inventory version 4.0 Generic core scales in healthy and patient populations. Med Care 2001, 39:800-812.

24. Varni JW, Seid M, Knight TS, Uzark K, Szer IS: The PedsQL 4.0 Generic core scales: sensitivity, responsiveness, and impact on clinical decisionmaking. J Behav Med 2002, 25:175-193.

25. Landgraf JM, Abetz L, Ware JE: The CHQ User's Manual. Boston: The Health Institute, New England Medical Center; 1996.

26. Engelen V, Haentjens MM, Detmar SB, Koopman HM, Grootenhuis MA: Health related quality of life of Dutch children: psychometric properties of the PedsQL in the Netherlands. BMC Pediatr 2009, 9:68.

27. Raat H, Landgraf JM, Bonsel GJ, Gemke RJBJ, Essink-Bot ML: Reliability and validity of the child health questionnaire-child form (CHQ-CF87) in a Dutch adolescent population. Qual Life Res 2002, 11:575-581.

28. Mendelson BK, Mendelson MJ, White DR: Body-esteem scale for adolescents and adults. J Pers Assess 2001, 76:90-106

29. Nakagawa S, Cuthill IC: Effect size, confidence interval and statistical significance: a practical guide for biologists. Biol Rev Camb Philos Soc 2007, 82:591-605.

30. Cohen J: Statistical power analysis. Current directions in psychological science 1[3]. New York: Association for Psychological Science; 1992:98-101.
31. Hofsteenge GH, Chinapaw MJM, Delemarre-van de Waal HA, Weijs PJM: Long-term effect of the Go4it group treatment for obese adolescents: a randomised controlled trial. Clin Nutr 2013. In press.

32. De Beer M, Hofsteenge GH, Koot HM, Hirasing RA, Delemarre-van de Waal HA, Gemke RJ: Health-related-quality-of-life in obese adolescents is decreased and inversely related to BMI. Acta Paediatr 2007, 96:710-714.

33. Varni JW, Limbers CA, Burwinkle TM: Impaired health-related quality of life in children and adolescents with chronic conditions: a comparative analysis of 10 disease clusters and 33 disease categories/severities utilizing the PedsQL 4.0 Generic core scales. Health Qual Life Outcomes 2007, 5:43.

34. Van Vlierberghe L, Braet C, Goossens L, Mels S: Psychiatric disorders and symptom severity in referred versus non-referred overweight children and adolescents. Eur Child Adolesc Psychiatry 2009, 18:164-173.

35. Steinsbekk S, Jozefiak T, Odegard R, Wichstrom L: Impaired parent-reported quality of life in treatment-seeking children with obesity is mediated by high levels of psychopathology. Qual Life Res 2009, 18:1159-1167.

36. Jelalian E, Mehlenbeck R, Lloyd-Richardson EE, Birmaher V, Wing RR: 'Adventure therapy' combined with cognitive-behavioral treatment for overweight adolescents. Int J Obes (Lond) 2006, 30:31-39.

37. Saelens BE, Sallis JF, Wilfley DE, Patrick K, Cella JA, Buchta R: Behavioral weight control for overweight adolescents initiated in primary care. Obes Res 2002, 10:22-32.

38. Wadden TA, Stunkard AJ, Rich L, Rubin CJ, Sweidel G, McKinney S: Obesity in black adolescent girls: a controlled clinical trial of treatment by diet, behavior modification, and parental support. Pediatrics 1990, 85:345-352.

39. Williamson DA, Martin PD, White MA, Newton R, Walden H, York-Crowe E, et al: Efficacy of an internet-based behavioral weight loss program for overweight adolescent African-American girls. Eat Weight Disord 2005, 10:193-203.

doi:10.1186/1471-2458-13-939

Cite this article as: Hofsteenge et al:: Effect of the Go4it multidisciplinary group treatment for obese adolescents on health related quality of life: a randomised controlled trial. BMC Public Health 2013 13:939.

\section{Submit your next manuscript to BioMed Central and take full advantage of:}

- Convenient online submission

- Thorough peer review

- No space constraints or color figure charges

- Immediate publication on acceptance

- Inclusion in PubMed, CAS, Scopus and Google Scholar

- Research which is freely available for redistribution 